\title{
Calcitonin gene related peptide in migraine: current therapeutics, future implications and potential off- target effects
}

\author{
Jason Charles Ray (1) ,,2 Mahima Kapoor, ${ }^{1,2}$ Richard J Stark, ${ }^{1,2}$ Shuu-Jiun Wang, ${ }^{3,4}$ \\ Lars Bendtsen, ${ }^{5,6}$ Manjit Matharu, ${ }^{7}$ Elspeth Jane Hutton (i) 1,2
}

- Additional material is published online only. To view please visit the journal online (http://dx.doi.org/10.1136/ jnnp-2020-324674)

'Neurology, Alfred Health, Melbourne, Victoria, Australia 2Department of Neuroscience, Monash University, Clayton, Victoria, Australia

${ }^{3}$ The Neurological Institute, Taipei Veterans General Hospital Taipei, Taiwan

${ }^{4}$ Brain Research Center, National Yang-Ming University, Taipei, Taiwan

DDanish Headache Center, Department of Neurology, Rigshospitalet Glostrup, Glostrup, Denmark

${ }^{6}$ University of Copenhagen, Kobenhavn, Denmark Headache Group, UCL Institute of Neurology and The National Hospital for Neurology and Neurosurgery, London, UK

Correspondence to Dr Jason Charles Ray, Neurology, Alfred Health, Melbourne, VIC 3004, Australia; j.ray@alfred.org.au

Received 25 July 2020 Revised 17 November 2020 Accepted 16 December 2020 Published Online First 25 January 2021

Check for updates

(C) Author(s) (or their employer(s)) 2021. No commercial re-use. See rights and permissions. Published by BMJ.

To cite: Ray JC, Kapoor M

Stark RJ, et al. J Neurol

Neurosurg Psychiatry

2021:92:1325-1334.

\section{ABSTRACT}

Migraine is the second largest cause of years lost to disability globally among all diseases, with a worldwide prevalence over 1 billion. Despite the global burden of migraine, few classes of therapeutics have been specifically developed to combat migraine. After 30 years of translational research, calcitonin gene-related peptide (CGRP) inhibitors have emerged as a promising new tool in the prevention of migraine. Like all new therapeutics; however, we have limited real-world experience and CGRP has several known systemic actions that warrant consideration. This article provides a narrative review of the evidence for CGRP antagonists and summarises the known and potential side effects that should be considered.

\section{INTRODUCTION}

Migraine is a common, potentially disabling disorder characterised by episodic attacks of moderate to severe headache, with a variety of neurological and systemic manifestations including photophobia, phonophobia, cutaneous allodynia, nausea, cognitive impairment and fatigue. It is classified by the International Classification of Headache Disorders 3 (ICHD-3) diagnostic criteria (table 1) as occurring with or without aura, and as either episodic or chronic (headache for $\geq 15$ days/month for 3 months, of which at least 8 days/month had features of migraine). ${ }^{1}$

Migraine has a strong female predominance $(3: 1)$, with a prevalence that peaks in the most productive years of life (ages 25-55). ${ }^{2}$ The worldwide prevalence is over 1 billion people, it is the second leading cause of years lived with disability overall, and the most common cause in people under 50 years. $^{34}$

Calcitonin gene-related peptide (CGRP) inhibitors represent one of only a few classes of medications developed specifically for migraine, the remainder having been co-opted from other indications with varying efficacy, and significant sideeffects that limit adherence.

\section{Methodology}

A narrative review was performed by searching electronic databases (Medline, Pubmed) using a variety of search terms such as 'CGRP and migraine' or 'CGRP and hypertension'. Owing to the wide variety of literature spanning multiple decades, we supplemented electronic searches with extensive eclectic searching using references lists, review articles and suggestive applications in PubMed (ie, similar articles).

\section{Pathophysiology of migraine}

Our understanding of migraine has expanded significantly over the last two decades. Current theories, of a disorder of sensory processing with multiple contributory genetic and hormonal factors are reviewed in depth elsewhere. ${ }^{6}$

\section{Prodrome and aura}

The earliest clinical phase of migraine is referred to as the prodromal phase and includes disturbances with concentration, fatigue, yawning, neck stiffness, depression and irritability which have been attributed to hypothalamic activation on functional imaging. $^{7-9}$

One-third of patients will experience transient neurological symptoms referred to as 'aura'. Migraine aura likely relates to a transient spreading wave of depolarisation of cortical neurons. Although not directly demonstrated, the phenotypic description and indirect imaging supports this hypothesis. ${ }^{610}$

\section{Headache phase}

The trigger of pain is still debated. In patients with aura, cortical spreading depression can act peripherally, activating Panx1 channels which results in sensitisation of afferent trigeminovascular fibres. These terminals that innervate the dura contain vasoactive neuropeptides including CGRP, substance $\mathrm{P}$, neurokinin $\mathrm{A}$ and pituitary adenylate cyclaseactivating peptide (PACAP) which are thought to be released when activated. Furthermore, two distinct functional networks that connect the cortex and the trigeminocervical complex (TCC) have been shown with tract tracing. The first arises from the insula and projects to lamina I and II neurons in the TCC and regulates trigeminovascular nociceptive tone. The second inhibitory network runs from the primary sensory cortex and projects into TCC lamina III and IV. Disruption of these networks may play a direct role in TCC activation. ${ }^{6}$

The trigeminal ganglion, which is involved in pain signalling and vascular dilatation, appears to be a pivotal structure. 5-hydroxytryptamine (serotonin) 


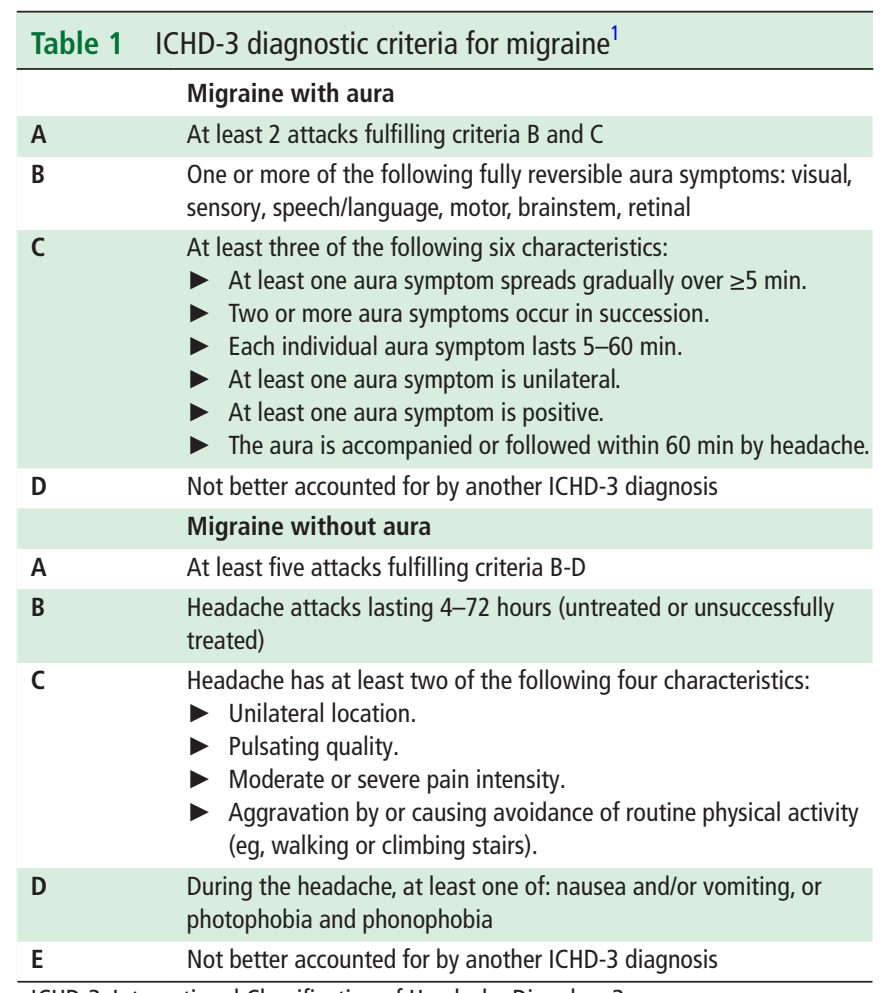

ICHD-3, International Classification of Headache Disorders-3.

receptors are abundant in its neurons and it has higher CGRPcontaining fibres and CGRP mRNA than other regions. ${ }^{11-14}$ Furthermore, the lack of a blood brain barrier makes it a potential therapeutic target. ${ }^{15}$

Nociceptive signalling from cranio-vascular structures is relayed via the TCC, which has been shown on functional imaging to activate ascending connections to other areas of the brain. ${ }^{16}$ The TCC itself has reflex connection with the superior salivatory nucleus, which is stimulated either directly from the brainstem or the dura. ${ }^{6}$ The TCC then has a complex network of ascending connections within the brainstem with other medullary pontine nuclei, midbrain nuclei, the ventrolateral periaqueductal grey and the cuneiform nucleus, leading to activation and subsequent sensitisation of second and third order nociceptive trigeminovascular neurons. ${ }^{17} 18$ Finally, there is wider activation of diencephalic nuclei within the hypothalamus, thalamus and cortex which is thought to contribute to the autonomic, endocrine, cognitive and affective symptoms experienced throughout migraine episodes. 6

\section{Postdrome}

Symptoms of the postdrome have not been thoroughly investigated, and include fatigue, impaired concentration, disturbed mood and neck stiffness. It is not known whether these symptoms are continued from the prodrome phase, or appear de novo during or after the pain. ${ }^{6}$ While often related to medication effect, these symptoms occur at similar rates with placebo. ${ }^{19}$

\section{Calcitonin gene-related peptide}

CGRP is increased during migraine, dilates blood vessels and is involved in nociceptor signalling. ${ }^{20-22}$ Its release is triggered by activation of transient receptor potential cation channel subfamily $\mathrm{V}$ member 1 and transient receptor potential ankyrin 1 channels, in response to a variety of agonists, as well as by angiotensin and norepinephrine. ${ }^{23}$
CGRP is a 37 amino acid peptide with two isoforms ( $\alpha$-CGRP and $\beta$-CGRP) which differ by only three amino acids and are encoded by two distinct genes-CALC1 and CALC2 on chromosome $11 .{ }^{13}$ It is a blood-brain barrier impermeant neuropeptide that is expressed throughout the nervous system, and in high concentrations in the striatum, amygdala, thalamus, pineal gland, colliculi, trigeminal ganglion, trigeminal nucleus caudalis, cerebellum and cerebral cortex, ${ }^{24}$ as well as peripherally in nociceptors and the enteric nervous system. It has a serum half-life of 7-10 minutes, however the tissue half-life is unknown. ${ }^{25}$ Skin flare of 6 hours following CGRP injection suggests either slow tissue clearance or prolonged receptor activation. ${ }^{26}$

The CGRP receptor is a G protein-coupled receptor comprising three subunits: calcitonin-like receptor, receptor activity-modifying protein 1 (RAMP1) and receptor component protein. ${ }^{13}$

In addition to the nervous system, CGRP is also found within perivascular varicosities of smooth muscle cells, mesenteric and submucosal plexi within the digestive system, widely throughout the vascular system and also in fibres innervating the sino-atrial node and right atrium. ${ }^{27}$

\section{CGRP inhibition}

There are two classes of CGRP inhibitors-monoclonal antibodies (mabs) and small molecule antagonists (gepants). Four monoclonal antibodies have undergone phase II and III trial in patients with episodic and chronic migraine, with post-trial follow-up up to 5 years. Erenumab, which targets the CGRP receptor, and eptinezumab, fremanezumab and galcanezumab which bind to the ligand. The findings of the major trials are summarised in tables 2 and 3.

\section{Small molecule CGRP antagonists \\ Olcegepant and telcagepant}

Olcegepant was the first CGRP antagonist to progress to phase II study in 1999 following promise in preclinical trials. While poor bioavailability prevented further development, it had a favourable side effect profile, with transient paraesthesia in $7 \%$ of patients[s1].

Telcagepant was investigated as an acute treatment for migraine. In phase II and III trials, it was found to be superior to placebo at 2 hours. With episodic use, it had a favourable side effect profile with $4 \%-6 \%$ of patients reporting dry mouth, somnolence, dizziness, nausea and/or fatigue[s2], [s3]. When trialled at a daily dose for migraine prevention; however, $2.3 \%-4.5 \%$ of patients developed elevated transaminases, which resulted in discontinuation of the drug. No other systemic effects emerged with regular administration[s4].

Another small molecule CGRP antagonist which has been discontinued is MK-3207. MK-3207 underwent phase II study, which found a dose-dependent trend towards pain freedom[s5]. Research was discontinued in 2009; however, due to elevation of liver enzymes[s6], [s7]. Finally, BI44370 had one subject with a markedly elevated value of liver enzymes, and its development status is not known[s8].

\section{Current gepants in trial}

Atogepant

Atogepant, a small molecule oral CGRP antagonist, has completed a phase II and III study at a variety of doses in prevention of episodic migraine in patients with no history of medication overuse headache $(\mathrm{MOH})$. There were no significant adverse events reported, and unlike its predecessors, 
Table 2 CGRP inhibitor response rates in major preventative trials

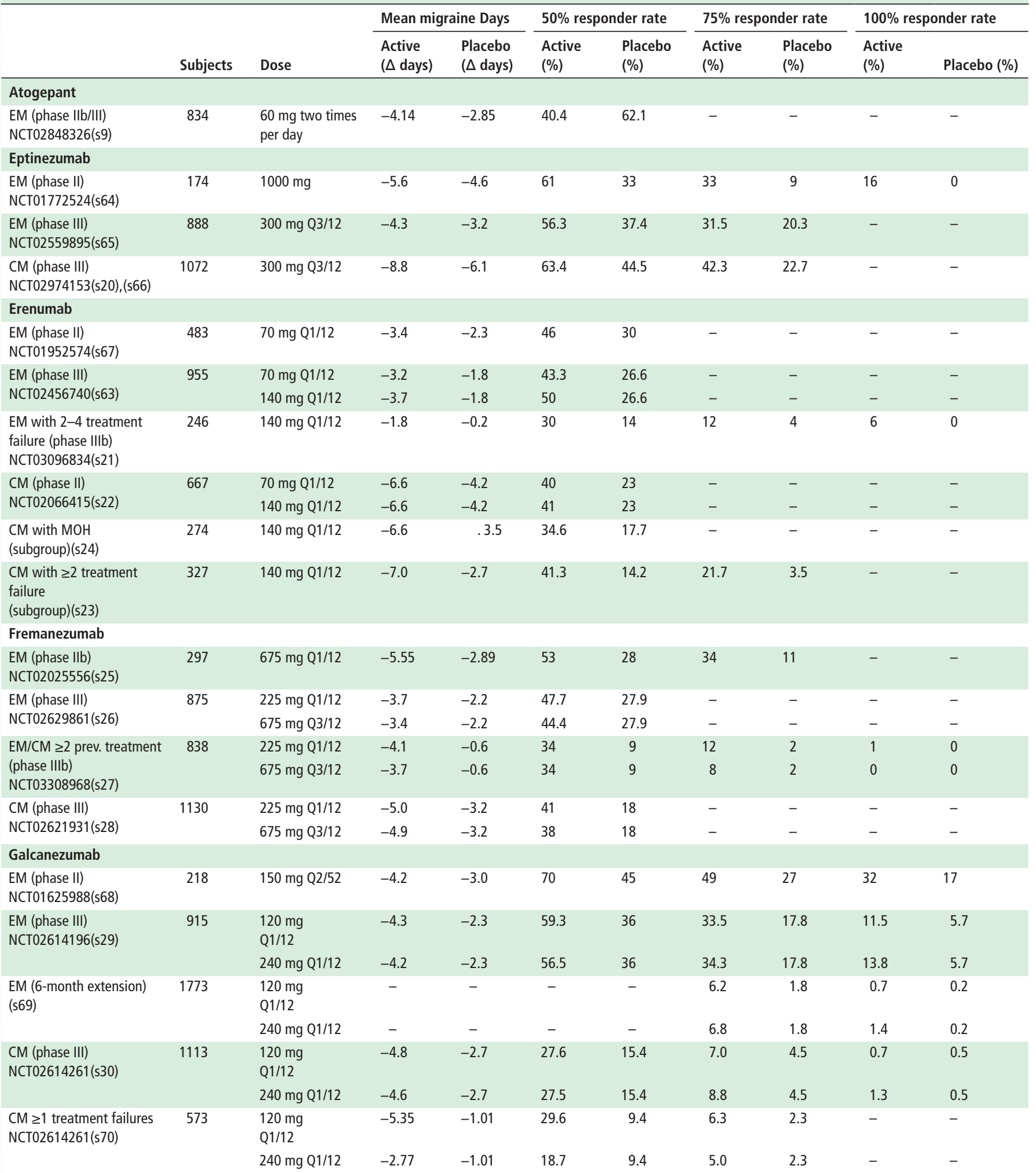

$\mathrm{CM}$, chronic migraine; $\mathrm{EM}$, episodic migraine; $\mathrm{MOH}$, medication overuse headache.

liver function remained normal[s9]. Further phase III studies are ongoing in both episodic (NCT03777059) and chronic (NCT03855137) migraine. Atogepant is not licensed under the US Food and Drug Administration (FDA), European Medicines Agency (EMA) or Australian Therapeutic Goods Administration (TGA)[s10]-[s12].
Rimegepant

Several phase IIb and III randomised control trials have been conducted investigating rimegepant in the acute treatment of migraine[s13]-[s15]. Pooled analysis has found that at a dose of $75 \mathrm{mg}, 2$ hours pain freedom was achieved for $20.6 \%$, compared with $12.5 \%$ in placebo. Adverse events were similar 
Table 3 CGRP inhibitor quality of life outcomes in major preventative trials

\begin{tabular}{|c|c|c|c|c|c|c|c|c|c|c|}
\hline \multirow{3}{*}{$\begin{array}{l}\text { Clinically significant } \\
\text { change }\end{array}$} & \multirow[b]{3}{*}{ Subjects } & \multirow[b]{3}{*}{ Dose } & \multirow{2}{*}{\multicolumn{2}{|c|}{$\begin{array}{l}\text { MIDAS } \\
-5(s 71)\end{array}$}} & \multirow{2}{*}{\multicolumn{2}{|c|}{$\begin{array}{l}\text { HIT-6 } \\
-2.5(s 64),(s 72)\end{array}$}} & \multirow{2}{*}{\multicolumn{2}{|c|}{$\begin{array}{l}\text { MSQ } \\
\text { R: 3.2, F: 7.5(s24) }\end{array}$}} & \multirow{2}{*}{\multicolumn{2}{|c|}{$\begin{array}{l}\text { MPFID } \\
3-5(s 63),(s 73)\end{array}$}} \\
\hline & & & & & & & & & & \\
\hline & & & Active & Placebo & Active & Placebo & Active & Placebo & Active & Placebo \\
\hline \multicolumn{11}{|l|}{ Eptinezumab } \\
\hline $\begin{array}{l}\text { EM (phase II) } \\
\text { NCT01772524(s64) }\end{array}$ & 174 & $1000 \mathrm{mg}$ & - & - & -10.1 & -7.7 & $\begin{array}{l}P:-28.5 \\
R:-21.4 \\
F:-23.1\end{array}$ & $\begin{array}{l}P:-22.2 \\
R:-18.0 \\
F:-21.1\end{array}$ & - & - \\
\hline \multicolumn{11}{|l|}{ Erenumab } \\
\hline \multirow[t]{2}{*}{$\begin{array}{l}\text { EM (phase III) } \\
\text { NCT02456740(s63) }\end{array}$} & 955 & $\begin{array}{l}70 \mathrm{mg} \\
\mathrm{Q} 1 / 12\end{array}$ & - & - & - & - & - & - & $\begin{array}{l}\text { A: }-5.5 \\
\mathrm{I}:-4.2\end{array}$ & $\begin{array}{l}\text { A: }-3.3 \\
\text { I: } 02.4\end{array}$ \\
\hline & & $\begin{array}{l}140 \mathrm{mg} \\
\mathrm{Q} 1 / 12\end{array}$ & - & - & - & - & - & - & $\begin{array}{l}\text { A: }-5.9 \\
\mathrm{I}:-4.8\end{array}$ & $\begin{array}{l}\text { A: }-3.3 \\
\mathrm{l}: 02.4\end{array}$ \\
\hline $\begin{array}{l}\text { EM with 2-4 treatment } \\
\text { failure (phase IIIb) } \\
\text { NCT03096834(s21) }\end{array}$ & 246 & $\begin{array}{l}140 \mathrm{mg} \\
\mathrm{Q} 1 / 12\end{array}$ & - & - & - & - & - & - & $\begin{array}{l}\text { A: }-3.4 \\
\text { I: }-1.9\end{array}$ & $\begin{array}{l}A:+0.6 \\
\mathrm{I}:+1.6\end{array}$ \\
\hline \multirow[t]{2}{*}{$\begin{array}{l}\mathrm{CM} \text { with } \mathrm{MOH} \\
\text { (subgroup)(s24) }\end{array}$} & 274 & $\begin{array}{l}70 \mathrm{mg} \\
\mathrm{Q} 1 / 12\end{array}$ & -22.0 & -3.6 & -5.2 & -2.9 & $\begin{array}{l}P:-11.6 \\
R:-17.1 \\
F:-17.1\end{array}$ & $\begin{array}{l}P:-7.7 \\
R:-11.7 \\
F:-8.2\end{array}$ & & \\
\hline & & $\begin{array}{l}140 \mathrm{mg} \\
\mathrm{Q} 1 / 12\end{array}$ & -16.1 & -3.6 & -5.4 & -2.9 & $\begin{array}{l}P:-10.5 \\
R:-17.4 \\
F:-15.9\end{array}$ & $\begin{array}{l}P:-7.7 \\
R:-11.7 \\
F:-8.2\end{array}$ & & \\
\hline \multicolumn{11}{|l|}{ Fremanezumab } \\
\hline $\begin{array}{l}\text { EM (phase Ilb) } \\
\text { NCT02025556(s25) }\end{array}$ & 297 & $\begin{array}{l}675 \mathrm{mg} \\
\mathrm{Q} 1 / 12\end{array}$ & -24.93 & -9.73 & & & & & & \\
\hline \multirow[t]{2}{*}{$\begin{array}{l}\text { EM (phase III) } \\
\text { NCT02629861(s26) }\end{array}$} & 875 & $\begin{array}{l}225 \mathrm{mg} \\
\mathrm{Q} 1 / 12\end{array}$ & -19.0 & -12.5 & & & & & & \\
\hline & & $\begin{array}{l}675 \mathrm{mg} \\
\mathrm{Q} 3 / 12\end{array}$ & -18.0 & -12.5 & & & & & & \\
\hline \multirow{2}{*}{$\begin{array}{l}\text { EM/CM } \geq 2 \text { prev. treatment } \\
\text { (phase IIIb) } \\
\text { NCT03308968(s27) }\end{array}$} & 838 & $\begin{array}{l}225 \mathrm{mg} \\
\mathrm{Q} 1 / 12\end{array}$ & -24.7 & -7.0 & -6.1 & -2.2 & -17.5 & -6.9 & & \\
\hline & & $\begin{array}{l}675 \mathrm{mg} \\
\mathrm{Q} 3 / 12\end{array}$ & -19.7 & -7.0 & -5.2 & -2.2 & -15.7 & -6.9 & & \\
\hline \multirow[t]{2}{*}{$\begin{array}{l}\text { CM (phase III) } \\
\text { NCT02621931(s28) }\end{array}$} & 1130 & $\begin{array}{l}225 \mathrm{mg} \\
\mathrm{Q} 1 / 12\end{array}$ & & & -6.8 & -4.5 & & & & \\
\hline & & $\begin{array}{l}675 \mathrm{mg} \\
\mathrm{Q} 3 / 12\end{array}$ & & & -6.4 & -4.5 & & & & \\
\hline \multicolumn{11}{|l|}{ Galcanezumab } \\
\hline \multirow[t]{2}{*}{$\begin{array}{l}\text { EM (phase III) } \\
\text { NCT02614196(s29) }\end{array}$} & 915 & $\begin{array}{l}120 \mathrm{mg} \\
\mathrm{Q} 1 / 12\end{array}$ & -21.2 & -12.0 & - & - & $\mathrm{R}:-28.5$ & $\mathrm{R}:-19.7$ & - & - \\
\hline & & $\begin{array}{l}240 \mathrm{mg} \\
\mathrm{Q} 1 / 12\end{array}$ & -20.2 & -12.0 & - & - & $R:-27$ & $\mathrm{R}:-19.7$ & - & - \\
\hline \multirow[t]{2}{*}{$\begin{array}{l}\text { CM (phase III) } \\
\text { NCT02614261(s30) }\end{array}$} & 1113 & $\begin{array}{l}120 \mathrm{mg} \\
\mathrm{Q} 1 / 12\end{array}$ & -20.3 & -11.5 & - & - & $\begin{array}{l}\mathrm{P}:-18.0 \\
\mathrm{R}:-21.8 \\
\mathrm{~F}:-21.0\end{array}$ & $\begin{array}{l}\mathrm{P}:-11.0 \\
\mathrm{R}:-16.8 \\
\mathrm{~F}:-14.1\end{array}$ & - & - \\
\hline & & $\begin{array}{l}240 \mathrm{mg} \\
\mathrm{Q} 1 / 12\end{array}$ & -17 & -11.5 & - & - & $\begin{array}{l}\mathrm{P}:-16.1 \\
\mathrm{R}:-23.1 \\
\mathrm{~F}:-20.7\end{array}$ & $\begin{array}{l}\mathrm{P}:-11.0 \\
\mathrm{R}:-16.8 \\
\mathrm{~F}:-14.1\end{array}$ & - & - \\
\hline \multirow[t]{2}{*}{$\begin{array}{l}\text { CM } \geq 1 \text { treatment failures } \\
\text { NCT02614261(s70) }\end{array}$} & 573 & $\begin{array}{l}120 \mathrm{mg} \\
\mathrm{Q} 1 / 12\end{array}$ & - & - & - & - & $\mathrm{R}:-21.6$ & $R:-13.6$ & - & - \\
\hline & & $\begin{array}{l}240 \mathrm{mg} \\
\mathrm{Q} 1 / 12\end{array}$ & - & - & - & - & $\mathrm{R}:-19.2$ & $R:-13.6$ & - & - \\
\hline
\end{tabular}

A, MPFID activities score; CM, chronic migraine; EM, episodic migraine; F, MSQ-functional score; I, MPFID physical impairment score; MPFID, Migraine Physical Function Impact Diary; MSQ, Migraine Specific Quality-of-life; P, MSQ-preventative score; R, MSQ-restrictive score.

in both groups, and included liver derangement (2.2\%), nausea $(1.6 \%)$, urinary tract infection $(1.5 \%)$ and dizziness $(0.8 \%)$ [s16]. Rimegepant is licensed under the FDA but not the EMA or TGA[s10]-[s12].

\section{Ubrogepant}

Ubrogepant has undergone phase II and III study in the acute treatment of migraine. One thousand six hundred and eighty-six patients were randomised to placebo, $25 \mathrm{mg}$ or $50 \mathrm{mg}$ of ubrogepant. The proportion of patients who experienced pain freedom at 2 hours was $14.3 \%, 20.7 \%$ and $21.8 \%$, respectively. The coprimary outcome, freedom of most bothersome symptom, was $27.4 \%$ in the placebo, $34.1 \%$ in the $25 \mathrm{mg}$ and $38.9 \%$ in the $50 \mathrm{mg}$ group[s17]. In 52-week open label extension, adverse events occurred in similar rates with placebo with the most common events reported including upper respiratory tract infection $(10.8 \%)$, sinusitis $(6.4 \%)$, nausea $(4.6 \%)$ and elevated transaminase (3.7\%). Liver derangement thus occurred 
in similar rates with placebo, and the only severe adverse event related to liver derangement normalised with treatment of cholecystitis[s18]. Ubrogepant is licensed under the FDA but not the EMA or TGA[s10]-[s12].

\section{Zavegepant}

Zavegepant (formerly vazegepant) is the first intranasal gepant, and is currently undergoing phase II/III study[s19]. We await the published results to see if local administration has similar efficacy and side effects compared with oral gepants.

\section{Monoclonal antibodies \\ Eptinezumab}

In phase II study, intravenous infusion of $1000 \mathrm{mg}$ of eptinezumab was trialled in patients aged $18-55$ with episodic migraine (mean migraine days $(\mathrm{MMD})=8.4 \pm 2.1$ ) excluding patients on other preventative medication, comorbid $\mathrm{MOH}$ or another headache type. Secondary outcomes of MMD and quality of life (QOL) numerically favoured eptinezumab but were not subjected to statistical analysis. No safety concerns were reported. Preliminary phase III trial of two doses of eptinezumab $(100 \mathrm{mg}$ or 300 mg every 12 weeks) has been reported and is positive (table 2) [s20]. Eptinezumab has been licensed under the FDA but not the EMA or TGA[s10]-[s12].

\section{Erenumab}

Erenumab has undergone phase III studies in both episodic and chronic migraine at $140 \mathrm{mg}$ subcutaneously 4 -weekly. The 50\% responder rate and change in MMD is detailed in table 2. In episodic migraine, the cohort of adult patients had mean MMD of $8.2 \pm 2.5$, and could continue preventative medication at a stable dose $(2.5 \%-3.1 \%$ patients, $55.2 \%-58.6 \%$ were treatment naïve). Exclusion criteria included recent use of onabotulinumtoxinA, device therapy, history of hemiplegic migraine, cluster headache or concurrent $\mathrm{MOH}$. In patients in the $70 \mathrm{mg}$ group, $43.3 \%$ had a $50 \%$ or greater reduction in MMD, while $50 \%$ of the $140 \mathrm{mg}$ saw a similar reduction (table 2), and saw a reduction in physical functional impairment scores (table 3). A further phase IIIb trial on patients with episodic migraine demonstrated efficacy of erenumab in patients who previously failed 2-4 preventative medications due to efficacy or tolerability[s21].

The phase II trial in chronic migraine had similar exclusion criteria, also excluding patients with continuous pain. Patients averaged 18.2 $\pm 4.7 \mathrm{MMD}$ and 21.1 $\pm 3.9 \mathrm{MHD}$, with $41 \%$ of patients suffering from $\mathrm{MOH}$ and $50 \%$ of the population having failed two or more preventative medications. Patients who received erenumab at either dose had a $6.6 \pm 0.4$ reduction in MMD compared with $4.2 \pm 0.4$ with placebo[s22]. In subgroup analysis of patients with previous treatment failure, similar reductions in MMD were seen (table 2) [s23].

A further subgroup analysis of erenumab in $\mathrm{CM}$ and $\mathrm{MOH}$ reported decreased MMD by 6.6 days in both the $70 \mathrm{mg}$ and $140 \mathrm{mg}$ groups, and improvements in QOL measured by HIT-6, Migraine Disability Assessment Scale (MIDAS), and the Migraine Specific Quality of life quesionnaire (MSQ) (tables 2 and 3)[s24]. Erenumab is licensed under the FDA, EMA and TGA[s10]-[s12].

\section{Fremanezumab}

Fremanezumab has undergone phase II and III study in episodic migraine administered subcutaneously at monthly (225 $\mathrm{mg})$ and quarterly $(675 \mathrm{mg})$ dosing regimens, recruiting patients on a stable dose of preventative medication, excluding patients on onabotulinumtoxinA. Despite being an exclusionary criterion,
$13 \%$ of patients reported medication overuse, and $27 \%$ had failed a previous preventative medication[s25], [s26]. In a combined phase IIIb study of patients with episodic and chronic migraine who had failed multiple medications (50\% failed 2, $30 \% 3 \%$ and 20\% failed four preventative medications), there was a reduction in number of migraine days of 3.7 with quarterly and 4.1 with monthly infusion compared with 0.6 days with placebo, and corresponding improvement in HIT-6 and MIDAS scores (table 3) [s27].

In phase III study of chronic migraine, patients had a baseline mean of $16.4 \pm 5.2$ migraine days/month, with up to $30 \%$ of the study population allowed to continue a stable dose of preventative medication. All other exclusion criteria were otherwise similar to other studies. Patients had $4.9 \pm 0.4$ and 5.0 \pm 0.4 reductions in MMD on quarterly and monthly infusion respectively, compared with a reduction of $3.2 \pm 0.4 \mathrm{MMD}$ with placebo[s28]. Fremanezumab is registered under the FDA, EMA and TGA[s10]-[s12].

\section{Galcanezumab}

Galcanezumab administered subcutaneously $240 \mathrm{mg}$ monthly was investigated in phase II and III trials of episodic migraine. Patients aged 18-65 who were not on another preventative medication or have comorbid $\mathrm{MOH}$ were recruited and had on average $9 \pm 2.9 \mathrm{MMD}$. Approximately $50 \%$ of patients had failed one previous preventative medication and $13.7 \%-15.3 \%$ having failed two or more[s29].

In phase III study in chronic migraine, similar exclusion criteria were employed; however, only patients with $>3$ preventative class failures were excluded. The study group had a mean of $19.6 \pm 4.6$ monthly headache days, $31.2 \%$ had failed $\geq 2$ preventative treatments and $64 \%$ had concurrent $\mathrm{MOH}$. The decrease in MIDAS score was not statistically significant, while MSQ scores were significantly lower (table 3)[s30]. In further study of patients who had failed multiple preventative medications (31.2\% failed $\geq 2,17.9 \%$ failed $\geq 3$ ), MSQ scores were significantly reduced.

Galcanezumab is currently undergoing study in episodic and chronic migraine in patients who have failed 2-4 preventative medications. Three-month data have been reported as a 4.1 reduction in MMD with galcanezumab compared with a reduction in MMD of 1.0 with placebo[s31]. Galcanezumab is registered under the FDA, EMA and TGA[s10]-[s12].

\section{CONCLUSION}

Overall, inhibition of CGRP for the treatment of migraine is biologically plausible and the evidence demonstrates significant reductions in headache days, with corresponding improvements of various QOL scores (that are largely comparable)[s32], [s33]. As noted elsewhere however, despite targeting a central neuropeptide, there remains a proportion of non-responders. Biomarker driven therapies would be of great help, but are hampered by CGRP's short half-life[s34].

There are several caveats that bear mentioning. First, the majority of phase III trials required patients to be taken off other preventives, excluded comorbid medication overuse (fremanezumab allowed $30 \%$ of recruited patients to continue one agent), and attempted to exclude patients who had failed multiple agents. Subsequent post-hoc analysis and phase IIIb trials appear equally efficacious in these more 'refractory' patients (table 2).

Finally, efficacy of CGRP inhibition in comparison to onabotulinumtoxinA therapy has not been assessed. There is preclinical data that CGRP and onabotulinumtoxinA may act differently 
on $\mathrm{C}$-fibres and $\mathrm{A} \delta$-fibres, suggesting possible synergistic benefit which has been described in small reviews, and warrants further investigation in refractory cases[s35]-[s37]. A European guideline for the use of CGRP inhibitors has been published, and is recommended to aid clinicians in their decision making[s38].

\section{Special populations}

Familial hemiplegic migraine

Infusion of CGRP in patients with known mutations of the familial hemiplegic migraine genes CACNA1A and ATP1A2 does not trigger a migraine attack ${ }^{22}$ [s39], [s40]. This suggests a separate pathophysiological process and raises questions about the efficacy of CGRP blockade in this cohort.

\section{Medication overuse}

Medication overuse is a frequent comorbidity in chronic migraine, with rates approaching 60\% in tertiary clinics[s41]. Given other highly efficacious treatments of migraine such as onabotulinumtoxinA have efficacy in $\mathrm{CM} / \mathrm{MOH}$ [s42], [s43], the utility of CGRP inhibition in this population is of particular relevance.

Biologically, the efficacy of CGRP inhibition is supported by animal studies. Opiate and triptan induced upregulation of CGRP has been demonstrated in neurons in the dorsal horn and dural afferents, respectively[s44]. Although incompletely understood, these findings suggest that $\mathrm{MOH}$ may be modulated by CGRP inhibition.

Of the CGRP monoclonal antibodies, erenumab alone has performed a subgroup analysis on patients with $\mathrm{MOH}$. In their phase III trial on chronic migraine, $41 \%$ of patients also met criteria for $\mathrm{MOH}$, and subgroup analysis demonstrated continued efficacy in this group of patients (table 2).

\section{Facial pain}

CGRP may also play a role in facial pain. CGRP receptors are expressed in the trigeminal nociceptive pathways presynaptically in A $\delta$-fibres as well as $40 \%$ of the neurons of the trigeminal ganglion, and post-synaptically[s45]-[s47]. Accordingly, CGRP has been found to be increased in trigeminal neuralgia patients[s48], [s49]. Furthermore, an association between CGRP levels and somatic, visceral, neuropathic and inflammatory pain suggests that CGRP may act as a neuromodulator in nonheadache conditions [s47].

The clinical features of trigeminal neuralgia are characterised by fast pain triggered by peripheral stimuli. A $\delta$-fibres are known to mediate the perception of pinprick (fast pain), while highthreshold neurons mainly respond to noxious mechanical stimuli. In rodent studies, fremanezumab has been shown to selectively inhibit high-threshold trigeminovascular neurons [s50]. In further study, fremanezumab inhibited A $\delta$-fibres but not C-fibres in the trigeminal ganglion[s51]. Taken together, it is likely that A $\delta$-fibres are involved in trigeminal neuralgia, and CGRP could play an important role in the hyper-excitability of the pathway[s47]. CGRP release from peripheral and central nerve endings from noxious stimulation is supportive of this[s52]. Thus, CGRP inhibition could be of benefit in trigeminal neuralgia by blocking CGRP in the trigeminal ganglion and/or in the primary sensory afferents in the root entry zone.

Clinical trials with erenumab (NCT04054024) and rimegepant (NCT03941834) for the treatment of trigeminal neuralgia are ongoing. Evidence of effect of CGRP inhibitors would be a major understanding of the pathophysiology of trigeminal neuralgia, and for the management of trigeminal neuralgia and possibly other neuropathic pain conditions.

\section{Cluster headache}

As detailed earlier, CGRP is widely expressed throughout the trigeminovascular system including the trigeminal ganglion, which has been shown to activate during cluster attacks[s53]. [s54]. Supporting the role of CGRP in cluster headache, activation of the trigeminal-autonomic reflex during attacks has been shown to release several vasoactive substances including CGRP, with elevated levels found in the ipsilateral jugular vein ictally[s55]. Finally, CGRP infusion has been shown to trigger attacks[s56]. The interaction between CGRP and cluster headache is summarised elsewhere[s55].

Galcanezumab has been studied in patients with episodic cluster headache, who were not taking other preventative therapy. Although halted early due to poor recruitment, the trial met its primary endpoint of reduced weekly frequency of attack at weeks one to three with a reduction of 8.7 headaches per week (71\% reduction) in patients who received galcanezumab compared with 5.2 headaches per week (50\% reduction) with placebo[s57]. When trialled in chronic cluster headache however, galcanezumab failed to meet its primary or secondary outcomes of reduction in headaches per week or percentage of patients with sustained reduction in headache[s58].

Fremanezumab has also been studied in cluster headache, however a phase III trial was stopped early as it was determined it would not meet its primary end-point of mean change from baseline in the monthly average number of cluster headache attacks during the 12-week treatment period.

A potential explanation for the conflicting results of CGRP inhibitors in cluster headache is a poor trial design. In one trial, patients needed 1 week of symptoms prior to randomisation, meaning they may have started to improve before the drug had an effect, and it would be impossible therefore to demonstrate efficacy. The role of CGRP in chronic cluster headache may also provide insight, with previous studies showing lower levels in chronic cluster headache compared with episodic cluster in remission[s59].

\section{Pregnancy and lactation}

There is no human data on the use of CGRP inhibitors during pregnancy or lactation. In pregnancy, animal studies did not show any evidence of harm, however CGRP does have a role in placental development and vascular adaptation with lower levels observed in pre-eclampsia. Blockade may affect the risk of pre-eclampsia, placental function and foetal weight[s60], [s61]. In lactation, there is a low transfer of $\operatorname{IgG}$ in milk and limited uptake in the gut. Given the theoretical risks and lack of evidence, continuation during pregnancy is not recommended; however, if required, may be considered in breast feeding in discussion with the patient[s62].

\section{Side-effects and off-target effect}

Given the wide expression of CGRP throughout the body, side effects and off-target effects are of particular interest. ${ }^{27}$ The overall theme in the clinical trials is that they are very well tolerated; the most commonly reported adverse events are constipation in $1.6 \%-3.4 \%$ of the cohort, nausea, local injection site reaction (5\%) and fatigue in $3 \%-6 \%$ of patients[s27], [s30], [s63].

Emerging real-world data, however, shows constipation occurs at a rate of $10 \%-20 \%$, with local site reactions, pruritus, bloating 


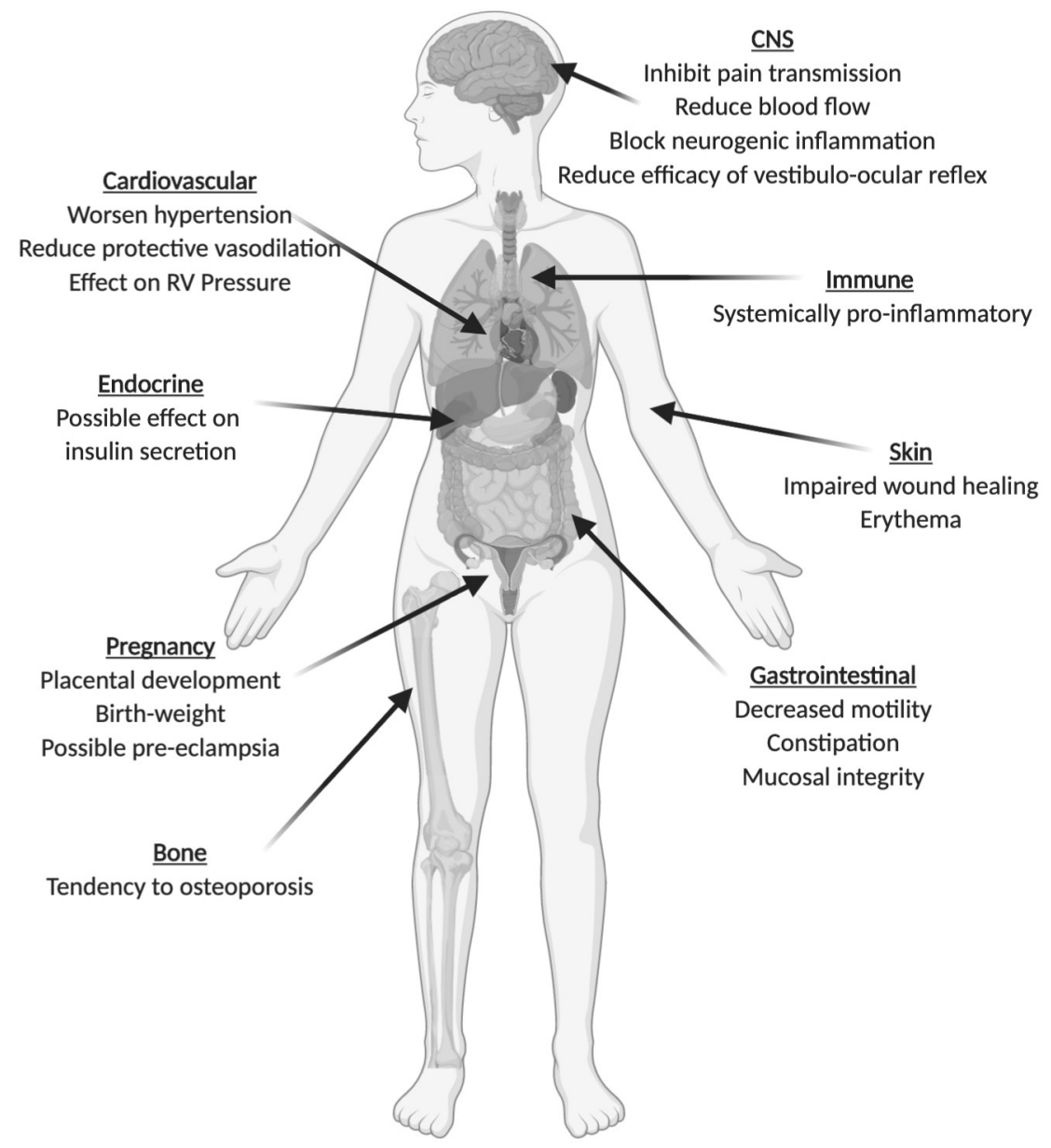

Figure 1 Possible systemic effects of calcitonin gene-related peptide inhibition.

or coryzal symptoms occurring in $1 \%-2 \%$ of patients. ${ }^{28-30}$ Reported events to the FDA included hair-loss, muscle cramps, hypersensitivity and cardiac complaints-palpitations $(n=80)$, tachycardia $(n=60)$, loss of consciousness $(n=27)$ and cardiac arrest $(n=5){ }^{30}$

The reason for the disconnect between CGRP action and reported side-effects is unclear. It may represent an internal redundancy in systemic compensatory mechanisms in important actions such as vasodilation, or the variable depth of gene expression in various body systems (with higher levels seen in the CNS (central nervous system) and gastrointestinal system). ${ }^{31}$ It is particularly noted that in retrospective review of novel therapeutics post FDA licensing between 2001 and 2010 and 32\% of drugs have a post-market safety event, at a median of 4.2 years (2.5-6.0). ${ }^{32}$ It remains imperative therefore, for the prescribing clinician to have an awareness of and vigilance for possible offtarget side effects. The possible systemic effects of inhibition of CGRP are discussed later, and summarised in figure 1.

\section{Neurological}

As a large molecule, $0.1 \%-1 \%$ of the drug will penetrate the blood-brain barrier, so action within the CNS is unlikely to be clinically relevant; however, it would be able to reach structures outside the blood-brain barrier, including the anterior pituitary, area postrema, choroid plexus and pineal gland. While CGRP receptors are found in the anterior pituitary, their role is not clear and thus care should be considered in their blockade in particular patients such as adolescents, and those with existing pituitary dysfunction. ${ }^{33}$ The impact of CGRP intracerebrally and on the blood-brain barrier is discussed elsewhere, however it may have a role in strengthening the blood-brain barrier and protecting the immune privilege of the brain. ${ }^{34}$

\section{Vestibular system}

CGRP immunoreactive neurons are also found both in efferent vestibular nuclei and peripherally in the vestibular system. ${ }^{35-37}$ Interestingly, from animal studies, CGRP appears to play a role in the function of the vestibular system. It is elevated in motion sickness, however loss of $\alpha$-CGRP reduces the efficacy of the vestibulo-ocular reflex. ${ }^{35} 36$ Whether inhibition of CGRP therefore provides an additional benefit in treatment of migraine-associated nausea beyond other therapeutics or impacts vestibular symptoms, warrants further investigation.

\section{Gastrointestinal}

Blockade of CGRP affects not only the CGRP receptors of the CNS, but also the CGRP receptors which predominate in the enteric nervous system. Blockade of CGRP has a dose dependent effect on motility in animal studies and this may explain why patients frequently experience constipation with these drugs. ${ }^{38}$

Animal studies suggest that CGRP also plays a role in mucosal integrity, with blockade of CGRP resulting in mucosal breakdown. ${ }^{39}$ Consideration should be given to this in patients suffering from peptic ulcer or inflammatory bowel disease. 


\section{Cardiovascular}

CGRP is now recognised as a potent microvascular vasodilator both through peripheral and possibly the RAMP1 component of CGRP receptor in the brainstem. ${ }^{40}$ There is growing evidence that while CGRP is not involved in the physiological control of blood pressure, it has an important protective role against the development of hypertension. ${ }^{41}$ Following the development and worsening of hypertension post-market, erenumab has added this as a warning label with the FDA.

Through similar mechanisms, CGRP also appears to have a role in the development of pulmonary artery hypertension (PAH). There is the suggestion of CGRP as a compensatory mechanism for $\mathrm{PAH}$, with higher levels observed in patients with more severe disease and infusion of CGRP producing a reduction of right ventricular pressure, hypertrophy and vascular remodelling. ${ }^{42}$

As a potent vasodilator, CGRP also has a protective role during coronary ischaemia, ${ }^{43}$ compounded by the association between migraine and coronary microvascular dysfunction independent of traditional vascular risk factors. ${ }^{44}$ Two trials have been performed to address the question of cardiovascular effects of CGRP inhibition. In the first, a single dose of erenumab was administered to 45 patients with stable angina who then underwent an exercise test. The trial participants did not demonstrate any change in angina frequency, exercise tolerance or mortality at 12 weeks. ${ }^{45}$ In the second, a supratherapeutic dose of telcagepant was given to 60 patients with stable angina. No alteration in treadmill exercise time was observed. ${ }^{46}$ Potential limitations of these studies include that the exercise testing was performed on day one after a single dose, which may not correspond to regular use, or long-term blockade. As discussed later, CGRP is elevated in exercise and may have a role in lipolysis. Whether long-term blockade impairs exercise tolerance, or may lead to weight gain is unknown. Finally, the generalisability of these findings to migraineurs, who may have microvascular dysfunction, remains unknown.

There is evidence for CGRP to have a neuroprotective role after ischaemic stroke by increasing blood flow, with CGRP administration poststroke in rats showing reduced post stroke oedema. ${ }^{47}$ In sub-arachnoid haemorrhage, elevated CGRP levels are seen in patients with more severe vasospasm suggesting a counter-regulatory process akin to those seen in PAH. This is further supported by post-mortem data showing patients who died from SAH had selective depletion of CGRP compared with other neuronal messengers, and infusion of CGRP which induced normalisation of cerebrovascular tone in $\mathrm{SAH}^{48}$

To date, there have been two cases reported in the literature involving vascular events occurring in patients receiving CGRP therapy. The first reported transient exercise-induced myocardial ischaemia in a patient who was administered sumatriptan 4 hours prior to the event. ${ }^{49}$ The second case was a middleaged woman who suffered a posterior circulation stroke while receiving CGRP therapy, a low dose contraceptive pill, and a triptan. ${ }^{50}$ One possible hypothesis suggested by the authors of the second case, that antagonism of CGRP-mediated vasodilation accentuated possible triptan-induced cerebral vasoconstriction requires further evaluation. ${ }^{51}$

\section{Endocrine/bone}

The effect of CGRP on insulin secretion is incompletely understood. There is contradictory evidence in preclinical studies as to whether CGRP stimulates, or has an inhibitory role on insulin secretion. ${ }^{52-55}$ The blockade of CGRP in animal models would suggest that CGRP inhibits insulin secretion and may shorten first-phase insulin secretion, however the effect was not large in a healthy animal model. ${ }^{56}$ CGRP levels are increased in obese female humans, and a murine knockout model showed improved glucose tolerance and insulin sensitivity, as well as higher metabolic rate and reduced body weight. ${ }^{23}$ CGRP is also elevated during exercise, and may have a role in lipolysis. ${ }^{57}$ The effect therefore of long-term blockade of CGRP, particularly in the context of patients with concurrent diabetes or insulinresistance, is not certain.

CGRP is considered an osteoanabolic peptide, and CGRP administration acts on osteoblast associated cells to stimulate osteoblast differentiation, as well as upregulate levels of activating transcription factor-4 and osteocalcin. Furthermore, CGRP activated osteoblasts also inhibit OPG/RANKL regulated osteoclastogenesis. ${ }^{58}$ The net effect of blockade of CGRP therefore, may be to preferentially promote osteoclast and downregulate osteoblast activity which may potentially lead to osteoporosis.

Conversely, a positive effect of CGRP inhibition may be seen in arthritis, where an increased level of CGRP in plasma and synovial fluid, as well as increased sensory innervation of the joint by CGRP positive fibres has been reported. Blockade of CGRP in animal models has demonstrated pain relief in osteoarthritis and reduced synovial proliferation in rheumatoid arthritis, suggestive of a possible therapeutic benefit in these groups. ${ }^{23}$

\section{Renal}

CGRP appears to have a reno-protective effect through regulation of blood pressure, and thus limitation of hypertensive related renal disease, a common cause of chronic renal disease. ${ }^{59}$ There is evidence however, that CGRP plays a role in the formation of fibrosis in the kidney, the underlying pathological process for all kidney disease. In animal models, denervation of the kidney reduced proinflammatory and profibrotic processes, and subsequent infusion of CGRP restarted these processes. ${ }^{60}$ The overall effect of long-term blockade of CGRP on kidney health therefore, is uncertain.

\section{Skin}

CGRP has an evident role in the skin, with skin flare seen following CGRP injection in adults. It follows that CGRP has a role in thermoregulation. Beyond this obvious association, CGRP also stimulates keratinocyte proliferation, migration and collagen maturation. When tested in rats CGRP was shown to be an important mediator of wound healing, with CGRP injection associated with significantly reduced wound closure times. ${ }^{61}$ Caution should therefore be employed in patients with baseline impairment of wound healing.

Similarly, CGRP modulates the cutaneous immune state. CGRP positive fibres are intimately associated with Langerhans cells in the epidermis, acting to reduce their antigen-presenting capacity, preventing unwanted inflammatory reactions and contributing to immunotolerance. The CGRP-induced shift in Langerhans cell profile from Th1 to Th2 may; however, be counteracted by a more inflammatory effect of CGRP inhibition on other immune cells. CGRP stimulates keratinocyte proliferation, but also their release of CGRP, interleukin $1 \beta$ (IL-1 $\beta$ ), IL-6 and tumour necrosis factor-alpha. ${ }^{62}$ Inhibition of CGRP may therefore have implications for condition such as psoriasis and atopic dermatitis, although the precise effect is hard to predict. 
Immunology

Systemically, CGRP is released both from immune and nerve cells, and acts directly on macrophages and dendritic cells to inhibit the production of inflammatory cytokines, as well as polarising T cells to a Th2 phenotype. Activation of the CGRP receptor increases cellular cAMP levels which leads to activation of protein kinase A, further mediating CGRP's anti-inflammatory effect. This effect may be exaggerated in mixed-bacterial sepsis, leading to immunosuppression and impaired host defence. ${ }^{63}$

Overall, therefore CGRP works as an inhibitive regulator of innate immune response, limiting tissue damage in inflammatory states. Prolonged antagonism of CGRP may therefore, lead to a pro-inflammatory state. The effect of this remains unknown, but long-term observation would be warranted given the role of inflammatory states in accelerated atherosclerosis. ${ }^{64} 65$

The impact of blockade of CGRP on overall immune balance in health and disease is difficult to predict, given the complexities of these multidirectional homoeostatic neuro-immune-endocrine interactions at both a tissue and systemic level. The potential immune impact of CGRP blocking therapeutics, particularly in the context of other immune therapies should be considered on a case by case basis, and closer monitoring may be warranted, particularly until there is greater experience in their use.

\section{Discussion of off-target effects of CGRP}

It is evident that there is a disconnect between the broad and encompassing systemic actions of CGRP described and the reported side-effects of the medications to date. There are several likely reasons for this. First, CGRP is only one regulatory peptide, and counter-regulatory processes may be accommodating its inhibition in the majority of patients. Second, it has been suggested that some of the reported adverse events to the FDA such as palpitations may relate to a normal physiological reaction to self-injection. Finally, as highlighted, some adverse events such as osteoporosis will not emerge in the short-term. Our review aims to summarise the possible adverse events for the clinician, so that they may be taken into consideration in risk-benefit decisions and safety monitoring (table 4).

\section{Future directions}

CGRP inhibitors represent an exciting development, as one of a few drug classes designed specifically for migraine. These medications provide an avenue for ongoing investigation to the pathology of migraine, and encouragement for further

Table 4 Checklist of disorders to monitor on calcitonin gene-related peptide inhibitor therapies

\begin{tabular}{lll}
\hline System & Possible benefit & Possible caution/consider \\
\hline Family history & SAH \\
Neurological & Osteoporosis \\
& Multiple sclerosis \\
Stroke
\end{tabular}

investigation of other neuro-peptides such as PACAP as therapeutic targets. ${ }^{66}$ There may also be a role for CGRP in treatment of other headache and pain disorders with co-morbid central sensitisation or neuroinflammatory mechanisms.

Nevertheless, the preclinical work describing CGRP and its multiple actions throughout the body raises the need for caution in certain patient populations when prescribing, and the need for long-term monitoring for potential late complications. Finally, further real-world data will be invaluable in determining how these medications compare to current therapies such as onabotulinumtoxinA, as well as the potential for synergistic use in addition to other preventative treatments.

See online supplemental file for online references[s1-s73].

Twitter Manjit Matharu @manjit_matharu and Elspeth Jane Hutton @spiraldance1

Contributors EJH and JCR were primarily responsible for study concept and design. JCR was responsible for review of literature and primary writing and preparation of the manuscript. MK provided substantial review and editing of manuscript. MM, LB, RJS and S-JW all provided additional expertise in review, editing and input into manuscript content.

Funding The authors have not declared a specific grant for this research from any funding agency in the public, commercial or not-for-profit sectors.

Competing interests MM serves on the advisory board for Allergan, Novartis, Eli Lilly, Autonomic Technologies and TEVA and has received payment for the development of educational presentations from Allergan, electroCore, Eli Lilly, Novartis and TEVA. RJS has been a member of Advisory Boards for Allergan, Novartis, Teva and Eli Lilly and has received Lecture Fees from Allergan, Novartis, Teva and Eli Lilly and Biogen. EJH has served on advisory boards for Sanofi-Genzyme, Novartis, Teva, Eli Lilly, Allergan, been involved in clinical trials sponsored by Novartis and Teva, and has received payment for educational presentations from Allergan, Teva, El Lilly and Novartis. S-JW has served on the advisory boards of Eli Lilly, Daiichi-Sankyo and Taiwan Norvatis. He has received honoraria as a moderator from Allergan, Pfizer, Eli Lilly, Bayer, and Eisai. He has received research grants from the Taiwan Minister of Technology and Science, Brain Research Center, National Yang-Ming University from The Featured Areas Research Center Programme within the framework of the Higher Education Sprout Project by the Ministry of Education (MOE) in Taiwan, Taipei Veterans General Hospital, and Taiwan Headache Society. LB serves on the advisory board for Allergan, Novartis, Eli Lilly and Teva, has been involved in clinical trials sponsored by Novartis and has received payment for educational presentations from Allergan, Teva and Novartis. JR and MK report no potential conflict of interest. Figure created with biorender.com.

\section{Patient consent for publication Not required.}

Supplemental material This content has been supplied by the author(s). It has not been vetted by BMJ Publishing Group Limited (BMJ) and may not have been peer-reviewed. Any opinions or recommendations discussed are solely those of the author(s) and are not endorsed by BMJ. BMJ disclaims all liability and responsibility arising from any reliance placed on the content. Where the content includes any translated material, BMJ does not warrant the accuracy and reliability of the translations (including but not limited to local regulations, clinical guidelines, terminology, drug names and drug dosages), and is not responsible for any error and/or omissions arising from translation and adaptation or otherwise.

\section{ORCID iDs}

Jason Charles Ray http://orcid.org/0000-0003-4833-5507

Elspeth Jane Hutton http://orcid.org/0000-0002-8543-7767

\section{REFERENCES}

1 International Headache Society. The International classification of headache disorders 3rd edition, 2019. Available: https://ichd-3.org/1-migraine/1-1-migraine-without-aura/ [Accessed 12 Aug 2019].

2 Lipton RB, Bigal ME. Migraine: epidemiology, impact, and risk factors for progression. Headache 2005:45 Suppl 1:S3-13.

3 James SL, Abate D, Abate KH, et al. Global, regional, and national incidence, prevalence, and years lived with disability for 354 diseases and injuries for 195 countries and territories, 1990-2017: a systematic analysis for the global burden of disease study 2017. The Lancet 2018;392:1789-858.

4 GBD 2016 Neurology Collaborators. Global, regional, and national burden of neurological disorders, 1990-2016: a systematic analysis for the global burden of disease study 2016. Lancet Neurol 2019;18:459-80.

5 Hepp Z, Dodick DW, Varon SF, et al. Adherence to oral migraine-preventive medications among patients with chronic migraine. Cephalalgia 2015:35:478-88.

6 Goadsby PJ, Holland PR, Martins-Oliveira M, et al. Pathophysiology of migraine: a disorder of sensory processing. Physiol Rev 2017:97:553-622. 
7 Argiolas A, Melis MR. The neuropharmacology of yawning. Eur J Pharmacol 1998:343:1-16

8 Denuelle M, Fabre N, Payoux P, et al. Hypothalamic activation in spontaneous migraine attacks. Headache 2007;47:070503104159006

9 Schulte LH, May A. The migraine generator revisited: continuous scanning of the migraine cycle over 30 days and three spontaneous attacks. Brain 2016;139:1987-93.

10 Cutrer FM, Sorensen AG, Weisskoff RM, et al. Perfusion-Weighted imaging defects during spontaneous migrainous aura. Ann Neurol 1998;43:25-31.

11 Weidner C, Klede M, Rukwied R, et al. Acute effects of substance P and calcitonin gene-related peptide in human skin--a microdialysis study. J Invest Dermatol 2000:115:1015-20.

12 Edvinsson L. The journey to establish CGRP as a migraine target: a retrospective view. Headache 2015;55:1249-55.

13 Yuan H, Lauritsen CG, Kaiser EA, et al. CGRP monoclonal antibodies for migraine: rationale and progress. BioDrugs 2017;31:487-501.

14 Rosenfeld MG, Mermod JJ, Amara SG, et al. Production of a novel neuropeptide encoded by the calcitonin gene via tissue-specific RNA processing. Nature 1983:304:129-35.

15 Eftekhari S, Salvatore CA, Johansson S, et al. Localization of CGRP, CGRP receptor, PACAP and glutamate in trigeminal ganglion. Relation to the blood-brain barrier. Brain Res 2015;1600:93-109.

16 Weiller C, May A, Limmroth V, et al. Brain stem activation in spontaneous human migraine attacks. Nat Med 1995;1:658-60.

17 Liu Y, Broman J, Zhang M, et al. Brainstem and thalamic projections from a craniovascular sensory nervous centre in the rostral cervical spinal dorsal horn of rats. Cephalalgia 2009:29:935-48.

18 Robert C, Bourgeais L, Arreto C-D, et al. Paraventricular hypothalamic regulation of trigeminovascular mechanisms involved in headaches. J Neurosci 2013:33:8827-40

19 Goadsby PJ, Dodick DW, Almas M, et al. Treatment-emergent CNS symptoms following triptan therapy are part of the attack. Cephalalgia 2007;27:254-62

20 Assas BM, Pennock JI, Miyan JA. Calcitonin gene-related peptide is a key neurotransmitter in the neuro-immune axis. Front Neurosci 2014:8:23.

21 Edvinsson L, Ekman R, Jansen I, et al. Calcitonin gene-related peptide and cerebral blood vessels: distribution and vasomotor effects. J Cereb Blood Flow Metab 1987;7:720-8.

22 Hansen JM, Hauge AW, Olesen J, et al. Calcitonin gene-related peptide triggers migraine-like attacks in patients with migraine with aura. Cephalalgia 2010:30:1179-86.

23 Russell FA, King R, Smillie SJ, et al. "Calcitonin gene-related peptide: physiology and pathophysiology," Physiological reviews, vol. 94, no. 4. American Physiological Society, pp. 1099-1142, 01-0ct-2014

24 Skofitsch G, Jacobowitz DM. Calcitonin gene-related peptide: detailed immunohistochemical distribution in the central nervous system. Peptides 1985:6:721-45.

25 Kraenzlin ME, Ch'ng JL, Mulderry PK, et al. Infusion of a novel peptide, calcitonin gene-related peptide (CGRP) in man. pharmacokinetics and effects on gastric acid secretion and on gastrointestinal hormones. Regul Pept 1985;10:189-97.

26 Brain SD, Williams TJ, Tippins JR, et al. Calcitonin gene-related peptide is a potent vasodilator. Nature 1985:313:54-6.

27 Poyner DR. Calcitonin gene-related peptide: multiple actions, multiple receptors. Pharmacol Ther 1992:56:23-51.

28 Ornello R, Casalena A, Frattale I, et al. Real-Life data on the efficacy and safety of erenumab in the Abruzzo region, central Italy. J Headache Pain 2020;21:32.

29 Ashina M, Goadsby PJ, Reuter U, et al. Long-Term safety and tolerability of erenumab: Three-plus year results from a five-year open-label extension study in episodic migraine. Cephalalgia 2019;39:1455-64.

30 Macdonald IAW, Gaigher I, Gaigher R. New drugs for flu, migraines. QuarterWatch, Inst safe Med Pract 2019.

31 Zeisel A, Hochgerner $\mathrm{H}$, Lönnerberg $\mathrm{P}$, et al. Molecular architecture of the mouse nervous system. Cell 2018;174:999-1014.

32 Downing NS, Shah ND, Aminawung JA, et al. Postmarket safety events among novel therapeutics Approved by the US food and drug administration between 2001 and 2010. JAMA 2017;317:1854

33 Wimalawansa SJ, el-Kholy AA. Comparative study of distribution and biochemical characterization of brain calcitonin gene-related peptide receptors in five different species. Neuroscience 1993;54:513-9.

34 Borkum JM. CGRP and brain functioning: cautions for migraine treatment. Headache 2019:59:1339-57.

35 Xiaocheng W, Zhaohui S, Junhui X, et al. Expression of calcitonin gene-related peptide in efferent vestibular system and vestibular nucleus in rats with motion sickness. PLoS One 2012; 7:e47308

36 Luebke AE, Holt JC, Jordan PM, et al. Loss of $\alpha$-calcitonin gene-related peptide $(\alpha C G R P)$ reduces the efficacy of the vestibulo-ocular reflex (VOR). J NeurosCi 2014:34:10453-8.
37 Popper P, Ishiyama A, Lopez I, et al. Calcitonin gene-related peptide and choline acetyltransferase colocalization in the human vestibular periphery. Audiol Neurootol 2002;7:298-302.

38 Bartho L, Koczan G, Maggi CA. Studies on the mechanism of the contractile action of rat calcitonin gene-related peptide and of capsaicin on the guinea-pig ileum: effect of hCGRP (8-37) and CGRP tachyphylaxis. Neuropeptides 1993;25:325-9.

39 Peskar BM, Wong HC, Walsh JH, et al. A monoclonal antibody to calcitonin generelated peptide abolishes capsaicin-induced gastroprotection. Eur J Pharmacol 1993;250:201-3.

40 Smillie S-J, Brain SD. Calcitonin gene-related peptide (CGRP) and its role in hypertension. Neuropeptides 2011;45:93-104.

41 Smillie S-J, King R, Kodji X, et al. An ongoing role of $\alpha$-calcitonin gene-related peptide as part of a protective network against hypertension, vascular hypertrophy, and oxidative stress. Hypertension 2014;63:1056-62.

42 Lo CCW, Moosavi SM, Bubb KJ. The regulation of pulmonary vascular tone by neuropeptides and the implications for pulmonary hypertension. Front Physiol 2018:9:1167.

43 MaassenVanDenBrink A, Meijer J, Villalón CM, et al. Wiping out CGRP: potential cardiovascular risks. Trends Pharmacol Sci 2016:37:779-88.

44 Aslan G, Sade LE, Yetis B, et al. Flow in the left anterior descending coronary artery in patients with migraine headache. Am J Cardiol 2013;112:1540-4.

45 Depre C, Antalik L, Starling A, et al. A randomized, double-blind, placebo-controlled study to evaluate the effect of Erenumab on exercise time during a treadmill test in patients with stable angina. Headache 2018:58:715-23.

46 Chaitman BR, Ho AP, Behm MO, et al. A randomized, placebo-controlled study of the effects of telcagepant on exercise time in patients with stable angina. Clin Pharmacol Ther 2012;91:459-66

47 Liu Z, Liu Q, Cai H, et al. Calcitonin gene-related peptide prevents blood-brain barrier injury and brain edema induced by focal cerebral ischemia reperfusion. Regul Pept 2011;171:19-25

48 Edvinsson L. Calcitonin gene-related peptide (CGRP) in cerebrovascular disease. ScientificWorldJournal 2002;2:1484-90.

49 Ashina M, Dodick D, Goadsby PJ, et al. Erenumab (AMG 334) in episodic migraine. Neurology 2017;89:1237-43.

50 Aradi S, Kaiser E, Cucchiara B. Ischemic stroke associated with calcitonin generelated peptide inhibitor therapy for migraine: a case report. J Stroke Cerebrovasc Dis 2019;28:104286

51 Amin FM, Asghar MS, Hougaard A, et al. Magnetic resonance angiography of intracranial and extracranial arteries in patients with spontaneous migraine without aura: a cross-sectional study. Lancet Neurol 2013;12:454-61.

52 Morishita T, Yamaguchi A, Yamatani T, et al. Effects of islet amyloid polypeptide (amylin) and calcitonin gene-related peptide (CGRP) on glucose metabolism in the rat. Diabetes Res Clin Pract 1992;15:63-9.

53 Rasmussen TN, Bersani M, Schmidt P, et al. Isolation and molecular characterization of porcine calcitonin gene-related peptide (CGRP) and its endocrine effects in the porcine pancreas. Pancreas 1998:16:195-204.

54 Seifert H, Sawchenko P, Chesnut J, et al. Receptor for calcitonin gene-related peptide: binding to exocrine pancreas mediates biological actions. Am J Physiol 1985;249:G147-51.

55 Leighton B, Cooper GJ. Pancreatic amylin and calcitonin gene-related peptide cause resistance to insulin in skeletal muscle in vitro. Nature 1988;335:632-5.

56 Tanaka H, Kashiwagi R, Koizumi T. Inhibition of calcitonin gene-related peptide (CGRP) has the potential to extend first-phase insulin secretion. Exp Clin Endocrinol Diabetes 2013;121:280-5.

57 Aveseh M, Koushkie-Jahromi M, Nemati J, et al. Serum calcitonin gene-related peptide facilitates adipose tissue lipolysis during exercise via PIPLC/IP3 pathways. Endocrine 2018:61:462-72.

58 He H, Chai J, Zhang S, et al. "CGRP may regulate bone metabolism through stimulating osteoblast differentiation and inhibiting osteoclast formation. Mol Med Rep 2016;13:3977-84.

59 Bowers MC, Katki KA, Rao A, et al. Role of calcitonin gene-related peptide in hypertension-induced renal damage. Hypertension 2005;46:51-7.

$60 \mathrm{Kim} \mathrm{J,} \mathrm{Padanilam} \mathrm{BJ.} \mathrm{Renal} \mathrm{denervation} \mathrm{prevents} \mathrm{long-term} \mathrm{sequelae} \mathrm{of} \mathrm{ischemic} \mathrm{renal}$ injury. Kidney Int 2015;87:350-8.

61 Khalil Z, Helme R. Sensory peptides as neuromodulators of wound healing in aged rats. J Gerontol A Biol Sci Med Sci 1996:51:B354-61.

62 Shi X, Wang L, Clark JD, et al. Keratinocytes express cytokines and nerve growth facto in response to neuropeptide activation of the ERK1/2 and JNK MAPK transcription pathways. Regul Pept 2013;186:92-103.

63 Holzmann B. Antiinflammatory activities of CGRP modulating innate immune responses in health and disease. Curr Protein Pept Sci 2013;14:268-74.

64 Hansson GK. Inflammation, atherosclerosis, and coronary artery disease. N Eng/ J Med 2005:352:1685-95.

65 Yudkin JS, Kumari M, Humphries SE, et al. Inflammation, obesity, stress and coronary heart disease: is interleukin-6 the link? Atherosclerosis 2000:148:209-14.

66 Edvinsson L, Tajti J, Szalárdy L, et al. PACAP and its role in primary headaches. J Headache Pain 2018;19:21. 